\title{
Influence of the Oxygen Partial Pressure on the Photo-Curing of Photo-Oxidation Induced Polycondensation Resin
}

\author{
Hisayoshi Arai $^{\dagger \ddagger}$, Yusuke Tajima ${ }^{\ddagger}$ and Kazuo Takeuchi ${ }^{\dagger \ddagger}$ \\ ${ }^{\dagger}$ Graduate School of Science and Engineering. Saitama University \\ 255 Shimoohkubo. Urawa-shi. Saitama 338-8570. Japan \\ ${ }^{\dagger}$ RIKEN (The Institute of Physical and Chemical Research) \\ 2-1 Hinosawa, Wako-shi. Saitama 351-0198, Japan
}

\begin{abstract}
The unique properties of the photo-oxidation induced polycondensation resin (POP resin) were experimentally investigated using ${ }^{1} \mathrm{H}$ NMR analysis, photolithographic evaluation and photo-DSC analysis. While a conventional photo-radial polymerization (PRP) type resin showed the phenomenon of reciprocity law failure at low intensity imadiation and decreasing thickness of the developed film with increased oxygen partial pressure, oxygen was found to improve the sensitivity of the POP resin Post-exposure baking (PEB) also enhanced the arring of the POP resin. The ${ }^{1} H$ NMR study indicated that the precursor at the crosslinking point is relatively stable at room temperature. The effectiveness of $\mathrm{C}_{60}$ added to the POP resin was discussed on the basis of the photo-DSC analysis.

Keywords: furan, fullerene, oxygen pressure, post-exposure baking
\end{abstract}

\section{Introduction}

Some furan derivatives such as furfuryl alcohol and methyl 2-furoate are easily oxidized and resinify upon exposure to air under light irradiation in the presence of an oxygen sensitizer $[1,2]$. Polymers having such a furan moiety in these side chains are expected to undergo crosslinking by singlet oxygen and yield a novel negative-type photopolymer, a photo-oxidation induced polycondensation resin (POP resin).

In typical photopolymers, dissolved oxygen molecules from the air tend to inhibit the curing reactions by quenching either the radicals or the excited initiators. Consequently, industrial photolithography requires preliminary steps to eliminate oxygen from the resist film. Either overcoating with PVA or purging by nitrogen is commonly used to remove oxygen [3]. On the other hand the POP resin uses the oxygen in its curing mechanism and is free from such unnecessary and complicated processes.

We have reported that oxygen sensitizers could be used as a photo-curing agent for a furan-substituted polymer, poly (2-furoic acid [2-(methacryloyloxy) ethyl] ester)(PFEMA) [4]. Among the sensitizers, fullerenes
$\mathrm{C}_{60}$ and $\mathrm{C}_{70}$ showed a superb ability to crosslink PFEMA under UV-irradiation $[5,6]$. This is due to the fact that fullerenes with the firm conjugated structures are able to better resist photo-breaching by oxidation than other sensitizers.

The POP resin has the following advantages over conventional photopolymers. First, the energy level of excitation is low $(22.5 \mathrm{kcal} / \mathrm{mol})$ [7], since singlet oxygen $\left({ }^{l} \Delta_{\mathrm{g}}\right)$ acts as the active species. It allows photolithography over a wide wavelength region from the ultraviolet to near infrared possible by choosing suitable sensitizers. Since fullerenes absorb light up to $700 \mathrm{~nm}$, the photo-curing proceeds even in the visible region. A second advantage is the fact that photo-curing in the POP resin is not hampered by oxygen molecules as discussed previously. Third, the polycondensation of oxygenated furans forms a firm conjugated structure. This guarantees the POP resin excellent thermo- and chemicalstabilities. Consequently, one of the promising fields of application for the POP resin is believed to lie in heat resistant photopolymers [8,9]. In order to clarify the unique properties of the POP type resin, we report in 
this paper the influence of the oxygen partial pressure during UV light irradiation of the poly-furfuryl methacrylate (PFMA) type POP resin containing fullerene [60], and compare the results with those for conventional photo-radial polymerization (PRP) type resists. The effect of post-exposure baking (PEB) is also investigated.<smiles>CC(C)CC1(C)CCCCC1(C)C(=O)OCc1ccco1</smiles><smiles>CC(=O)OCc1ccco1</smiles>

FAc<smiles>CC(=O)OCC12C=CC(OO1)O2</smiles>

FAc endoperoxide

\section{Experimental}

\subsection{Materials and Synthesis}

Fullerenes $\mathrm{C}_{60}$ was purchased from the Term $\mathrm{Co}$. and was $>\mathbf{9 9 . 9 8 \%}$ pure. Methyl methacrylate (MMA), furfuryl alcohol (FA), benzyl methacrylate (BzMA), 2,2-azobisisobutyronitrile (AIBN), diglyme and $o$-dichlorobenzene were obtained from the TCI Co., Ltd. MAX3510 as a multifunctional acrylate and IRG369 (Ciba Specialty Chemicals) as a photo-radical initiator were supplied by the JSR Co. FA was purified by vacuum distillation (bp. $65^{\circ} \mathrm{C}$, 11torr). AIBN was recrystallized in ethanol before use. All other reagents were used as received.

A furan-substituted methacryl monomer, furfuryl methacrylate (FMA), was obtained by the transesterification[10] of methyl methacrylate (MMA) using furfiyl alcohol (FA). As a catalyst, $\mathrm{Na}_{2} \mathrm{CO}_{3}(10 \mathrm{~g})$ was added to the liquid mixture of MMA (200g) and FA (200g). Methanol generated as a byproduct was eliminated from the reacting solution by fractional distillation while the solution was heated at $90^{\circ} \mathrm{C}$ for 24 hours. Next, the reacted mixture was washed with an aqueous solution of $1 \% \mathrm{NaOH}$. The separated organic phase was dried with anhydrous $\mathrm{MgSO}_{4}$ and then distilled under reduced pressure. Finally, FMA (bp. $82^{\circ} \mathrm{C}$, 5torr) was obtained in an yield $88 \%$. FT-IR (Perkin-Elmer Co.) and

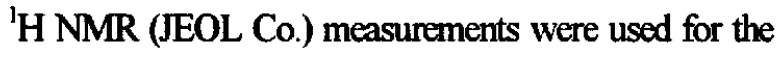
identification of the product.

Poly-furfuryl methacrylate (PFMA) was synthesized by the free radical polymerization of FMA(33wt\%) in $200 \mathrm{~g}$ of diglyme using an AIBN initiator $\left(2 \times 10^{-3} \mathrm{wt} \%\right.$ of FMA) at $80^{\circ} \mathrm{C}$ for 5.5 hours under a $\mathrm{N}_{2}$ atmosphere with stirning. Polymer conversion was determined by the total solid contents and found to be almost $100 \%$. To eliminate the remaining AIBN, the reacted solution was heated at $100^{\circ} \mathrm{C}$ for $30 \mathrm{~min}$ after polymerization. From the gel permeation chromatography (GPC) (Waters Co.) analysis, the $M_{n}$ and $M_{n} / M_{n}$ were determined to be $3.6 \times 10^{4}$ and 2.33 , respectively.

The PRP resin was prepared by the addition of MAX3510 and IRG369 (80 wt\% for the binder resin and $4 \mathrm{wt} \%$ for MAX3510) into the diglyme solution of poly-benzyl methacrylate (PBzMA) $\left(M_{n}=3.1 \times 10^{4}\right.$, $\left.M_{w} / M_{n}=2.24\right)$ as the binder resin. The solution was then diluted with diglyme for a total solid content of $20 \mathrm{wt} \%$.

\subsection{Lithographic evaluation using characteristic curves}

The resin prepared by dissolving PFMA and $\mathrm{C}_{60}$ in diglyme was spread onto a \#7059 glass substrate (Corning Co., Ltd.) using a spin-coater, and prebaked on a hot-plate at $100^{\circ} \mathrm{C}$ for $60 \mathrm{sec}$. The film thickness was fixed at $1.5 \mu \mathrm{m}$. It was then exposed to UV-vis radiation from a $500 \mathrm{~W}$ high pressure mercury lamp through an IR cut-off filter (IRA-25) (Toshiba), a deep UV cut filter (\#7059 glass) and a negative-type photo-mask (Toppan Printing Co., Ltd.). The light intensity was nonhomogeneous on the substrate ranging from 15.0 to $25.4 \mathrm{~mW} / \mathrm{cm}^{2}$ at $365 \mathrm{~nm}$. This spatial intensity variation on the substrate was used to check the reciprocity law. After irradiation, the film was baked on a hot plate for $60 \mathrm{sec}$. at $100^{\circ} \mathrm{C}$, and developed in the mixture of toluene $/$ methanol $=2 / 1$ for $60 \mathrm{sec}$.

The influence of the oxygen partial pressure was investigated as follows. The film coated substrate was allowed to stand for 3 hours in a glove box filled with the mixture of oxygen and nitrogen gases at latm of which the oxygen partial pressure was set between zero and 760 torr. It was then placed in a sealed $100 \mathrm{ml}$ cell equipped with a \#7059 glass window. After the cell was taken out of the glove box, the substrate was exposed to light through the window.

\subsection{Degradation of the furan endoperoxide}

The solution of toluene- $\mathrm{d}_{8}$ containing furfuryl acetate (FAc) $\left(1 \times 10^{-1} \mathrm{M}\right)$ and $\mathrm{C}_{70}\left(1 \times 10^{-3} \mathrm{M}\right)$ as a sensitizer was irradiated using a $500 \mathrm{~W}$ Xe arc lamp for $300 \mathrm{~min}$. at $-50^{\circ} \mathrm{C}$ in an NMR tube. After irradiation, the sample was kept at either $23.5,38.5,47.5$ or $57.5^{\circ} \mathrm{C}$ and 
the change in the ' $\mathrm{H}$ NMR spectrum of the generated furan acetate endoperoxide was measured with time.

2.4. Observation of the progress during the photoreaction using a photo-differential scanning calorimeter

A differential scanning calorimeter (DSC) (TA instruments 2920) was modified and used for the photo-DSC measurements. A known mass ( $7.70 \mathrm{mg})$ of a PFMA/C ${ }_{60}$ film $(\sim 20 \mu \mathrm{m}$ thick) was placed on a crimped aluminum sample pan equipped with a quartz window. The pan was then placed in a chamber which was purged with either dry oxygen or air during exposure. The UV radiation was guided to the sample and the reference in the pan through quartz windows, one mounted on the chamber and one on the pan. The samples were exposed to the unfiltered output of a $200 \mathrm{~W}$ high pressure mercury lamp mounted above the photo-DSC head. The light intensity was measured using an illuminometer and was fixed at 30.02 $\mathrm{mW} / \mathrm{cm}^{2}$.

\section{Results and Discussion}

\subsection{Selection of the solvent for $P O P$ resin}

In this POP resin, $\mathrm{C}_{60}$ was used as the sensitizer, PFMA as the furan ring modified polymer and diethylene glycol dimethyl ether (diglyme) as the solvent. $\mathrm{C}_{60}$ is known to be only slightly soluble in diglyme [11]. However, the diglyme solution of PFMA was found to easily dissolve $C_{60}$. The good affinity of $C_{60}$ to PFMA is believed to help $\mathrm{C}_{60}$ disperse in PFMA. The selection of solvents is also important for utilizing POP resin as a photopolymer. The molecules, which have an electron donating group, such as an amine, must be kept away from the reaction system, since it provides electrons to the excited $\mathrm{C}_{60}$ to easily yield the $\mathrm{C}_{60}$ anion which hampers the energy transfer from the triplet $\mathrm{C}_{60}$ to the oxygen molecule $[12,13]$. For example, the excessive addition of 1-methyl-2-pyridone (NMP) to the $\mathrm{C}_{60}$ solution in benzene shortened the lifetime of the $\mathrm{C}_{60}$ triplet from $27.5 \mu \mathrm{s}$ to $5.0 \mu \mathrm{s}$. When NMP was used as a solvent for the POP resin, the photo-curing was found to be experimentally inhibited due to residual NMP in the film.

\subsection{Effect of post-exposure baking on the lithogra- phy of POP resin}

The photo-curing characteristic of the POP resin was investigated using photolithography. Figure 1 shows the characteristic curves for the POP resin (25wt\% PFMA and $1 \times 10^{-3} \mathrm{M}$ of $\mathrm{C}_{60}$ in diglyme) irradiated in air and treated with or without PEB on a hot plate at $100^{\circ} \mathrm{C}$ for $60 \mathrm{sec}$. The curve shifted to the left when PEB was

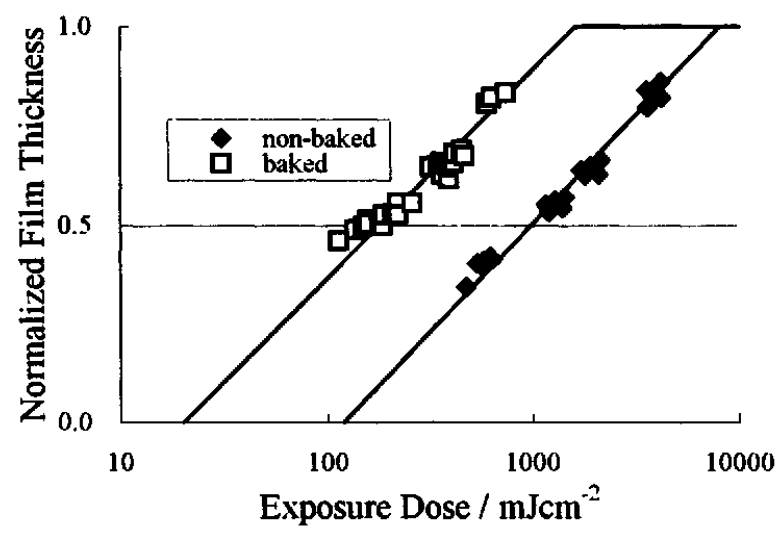

Figure 1 Characteristic curves of PFMA-C $\mathrm{C}_{60}$ resin with $\mathrm{PEB}$ at $100^{\circ} \mathrm{C}$ for $60 \mathrm{sec}$. $(\diamond)$ and without PEB ( $\square$ ). 20wt\% PFMA in diglyme with $1 \times 10^{-3} \mathrm{M}$ $\mathrm{C}_{60}$ was prebaked at $100^{\circ} \mathrm{C}$ for $60 \mathrm{sec}$. and developed with toluene for $60 \mathrm{sec}$. Light intensity is $25.7 \mathrm{~mW} / \mathrm{cm}^{2}$.

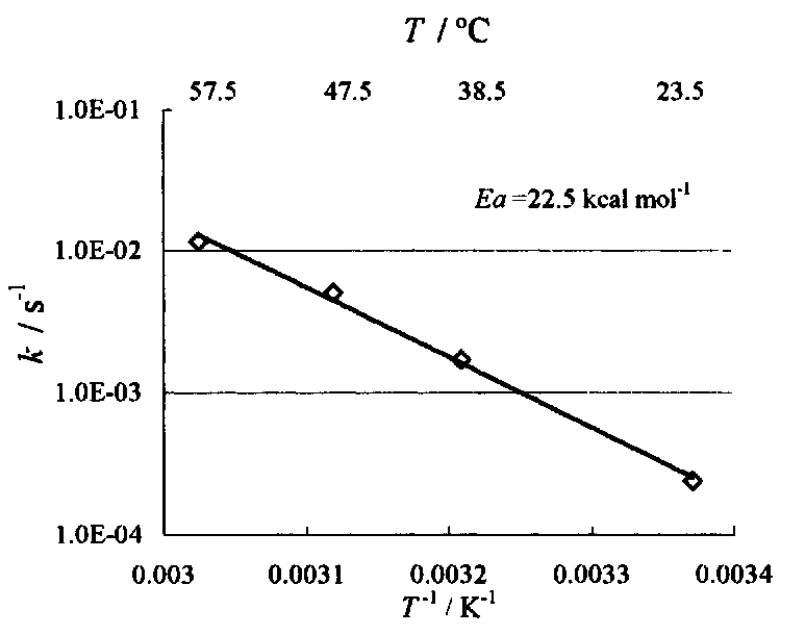

Figure 2 The activation energy for the degradation of FAc endoperoxide calculated from decreasing ' $H$ NMR peak at $\delta 4.39$ corresponding to methylene group.

adopted. This improved sensitivity is probably due to the fact that furan endoperoxide, the precursor at the crosslinking point, which is relatively stable at room temperature, is decomposed by PEB.

In order to study the thermostability of the furan endoperoxide, furfryl acetate (FAc) was used as a model compound. When the deuterated toluene solution of $F A c$ and $C_{70}$ was irradiated by the light of a $500 \mathrm{~W}$ $\mathrm{Xe}$ lamp at $-50^{\circ} \mathrm{C}$, the result of the 'H NMR measurement indicated the generation of the endoperoxide [14]. This intermediate is stable at low temperature, but the temporal ${ }^{1} H$ NMR spectrum of the irradiated sam- 


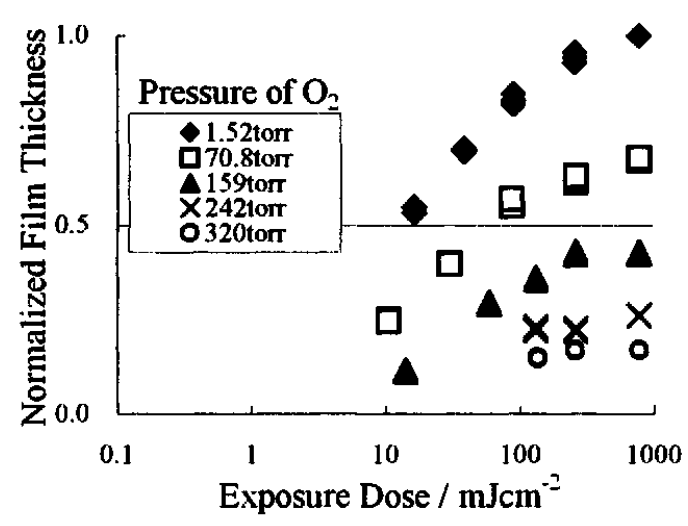

Figure 3 Characteristic curves of the conventional photo-radical polymerization resist, consisting 10wt\% PBzMA, 8wt\% IRG369 and $0.8 \mathrm{wt} \%$ MAX3510 in diglyme, for different partial pressures of oxygen. Resins were prebaked at $100^{\circ} \mathrm{C}$ for $60 \mathrm{sec}$, post- exposure baked at $100^{\circ} \mathrm{C}$ for $60 \mathrm{sec}$ and developed with toluene $/$ methanol $=2 / 1$ for $60 \mathrm{sec}$. Light intensity is $25.7 \mathrm{~mW} / \mathrm{cm}^{2}$.

ple at room temperature showed that it gradually thermally decomposed in a first-order reaction fashion. The apparent rate constant and the activation energy for the degradation of the FAc endoperoxide were estimated from the decreasing intensity of the ${ }^{1} \mathrm{H}$ NMR peak at $4.39(\mathrm{dd}, 2 \mathrm{H})$ corresponding to the methylene group. They were found to be $1.68 \times 10^{-2} \mathrm{~s}^{-1}$ at $60^{\circ} \mathrm{C}$ and $22.5 \mathrm{kcal} \mathrm{mol}^{-1}$, respectively (Figure 2 ). The relatively large activation energy for the degradation suggests that PEB accelerates the crosslinking formation. The lifetime of the endoperoxide $\left(60 \mathrm{sec}\right.$ at $60^{\circ} \mathrm{C}$ ) was considered as the minimum required time for $\mathrm{PEB}$.

\subsection{Effect of oxygen pressure}

Photolithography using the POP resin under various

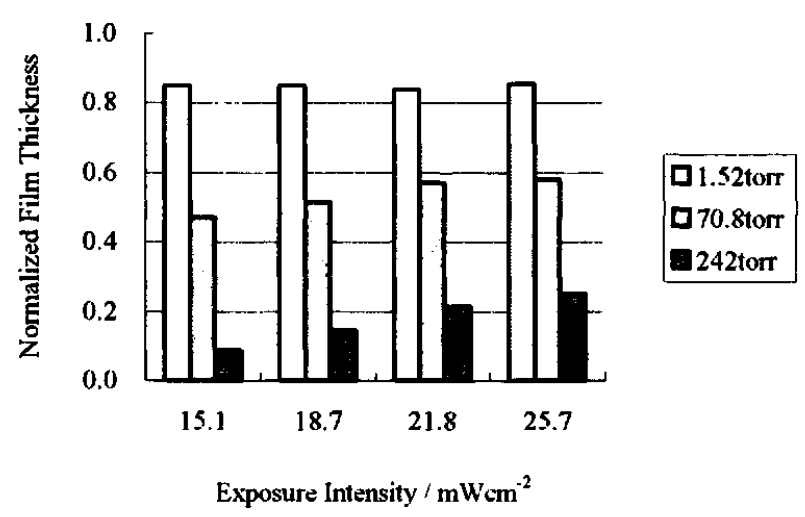

Figure 5 Exposure intensity dependence on developed film thickness of PBzMA / IRG369 / MAX3510 resin. Partial oxygen pressures are 1.52, 70.8 and 242torr. Exposure dose is $100 \mathrm{~mJ} \mathrm{~cm}^{-2}$.

oxygen pressures was performed and the sensitivity, contrast and remaining film thickness were studied and compared with those for the conventional PRP resin containing MAX3510, IRG369 and poly-benzyl methacrylate in diglyme. The characteristic curves of the PRP and POP resins are shown in Figure 3 and Figure 4 , respectively, where the mixing ratio of oxygen and nitrogen in the ambient gas at latm was changed from null (full nitrogen) to unity (full oxygen). The developed film thickness remarkably decreased with the increase in the partial pressure of oxygen for the PRP resin. The pattern formation became impossible at such high $\mathrm{O}_{2}$ pressures as 450 torr, although no change in the threshold exposure dose for the start of the insolubilization was observed. If the excited initiator had been directly inhibited by ambient $\mathrm{O}_{2}$, the threshold would have increased with $\mathrm{O}_{2}$ pressure. This shows that $\mathrm{O}_{2}$ hardly inhibits the excited initiator in this case. On the other hand, the saturating tendency with increasing dose

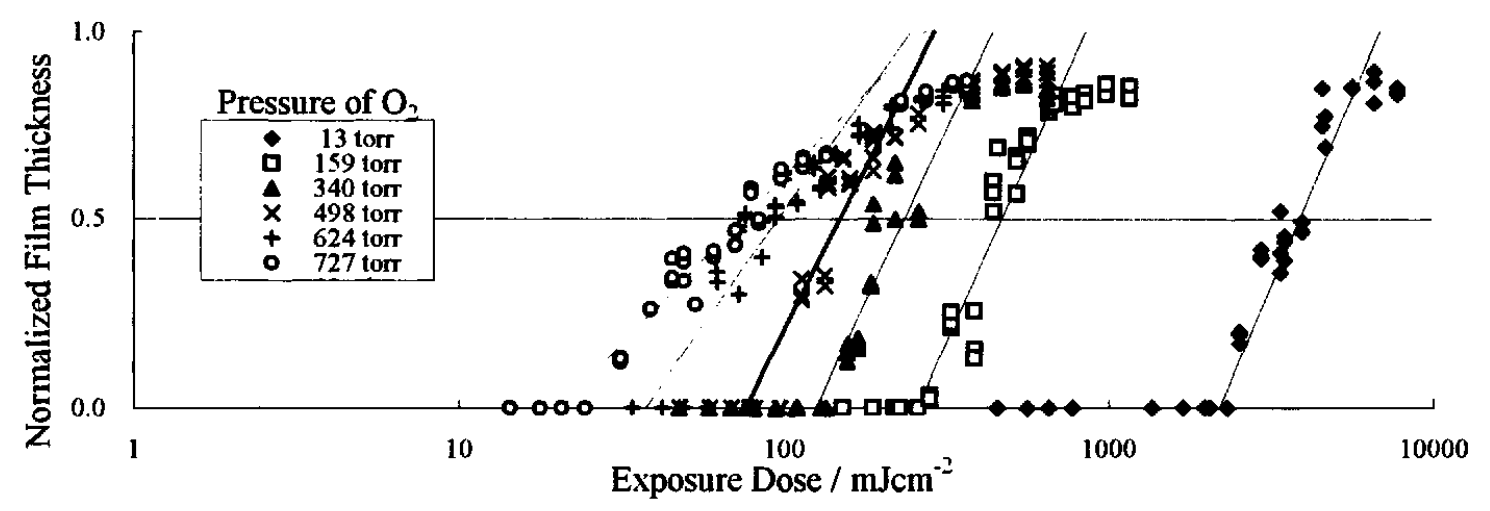

Figure 4 Characteristic curves of PFMA- $\mathrm{C}_{60}$ resin, $20 \mathrm{wt} \%$ PFMA in diglyme with $1 \times 10^{-3} \mathrm{M} \mathrm{C}_{60}$, for different partial pressure of oxygen. Resins were prebaked at $100^{\circ} \mathrm{C}$ for $60 \mathrm{sec}$, post-exposure baked at $100^{\circ} \mathrm{C}$ for $60 \mathrm{sec}$ and developed with toluene/methanol $=2 / 1$ for $60 \mathrm{sec}$. Light intensity is $25.7 \mathrm{~mW} / \mathrm{cm}^{2}$. 
Table 1 The sensitivities $S^{0.5}$ and contrasts $\gamma$ of PFMA-C 60 resin at different oxygen partial pressures from the results in Figure $3 \mathrm{~b}$.

\begin{tabular}{ccc}
\hline $\begin{array}{c}\text { Oxygen pressure } \\
p_{02} / \text { torr }\end{array}$ & $\begin{array}{c}\text { Sensitivity } \\
S^{0.5} / \mathrm{mJ} \mathrm{cm}^{-2}\end{array}$ & $\begin{array}{c}\text { Contrast } \\
r\end{array}$ \\
\hline 13 & 3860 & 2.03 \\
159 & 468 & 1.93 \\
340 & 234 & 1.84 \\
498 & 149 & 1.72 \\
624 & 96 & 1.23 \\
727 & 75 & 0.90 \\
\hline
\end{tabular}

in Figure 3 suggests that the propagation reaction of polymerization is inhibited. It is reasonable assume that $\mathrm{O}_{2}$ does quench radicals.

At the high oxygen pressures, the reciprocity law failure at a low intensity was observed as shown in Figure 5. This is due to the following reason. When the generation rate of the radicals is low at a low intensity, oxygen abundant in the film effectively quenches the radicals. On the other hand, when the intensity is high, the oxygen concentration is lowered after it is consumed by excess generated radicals. Since the $\mathrm{O}_{2}$ diffusion from the air is slow, the $\mathrm{O}_{2}$ concentration does not quickly recover. Therefore, inhibition by oxygen in the latter stage is not as serious as in the former case.

In contrast to the case for the PRP resin, the POP resin showed improved sensitivity with increased oxygen pressure without loss of thickness in the developed films (Table 1). Since no inhibitor for singlet oxygen is present under this experimental condition, no reciprocity law failure was observed in the experiment at low intensity, although a possible deviation from reciprocity law is not ruled out at high intensity with excessive $\mathrm{C}_{60}$ in the polymer, due to the biphotonic triplet-triplet anmihilation process, as observed in many cases using laser flash photolysis[15].

The influence of the concentration of oxygen in a polymer film on the reaction rate was also studied by photo-differential scanning calorimetry (photo-DSC). The photo-DSC chart for PFMA containing $\mathrm{C}_{61}$ is illustrated in Figure 6. It was also confirmed by this method that the photoreaction in the flowing oxygen gas proceeds faster than that in flowing air.

In the conventional PRP resin, the exotherm has a sharp peak, and the heat flow decreases toward null with increasing time as the initiator is consumed [16]. In our case for the POP resin, the exotherm monotonically increases. This suggests that $\mathrm{C}_{60}$ maintains the ability to

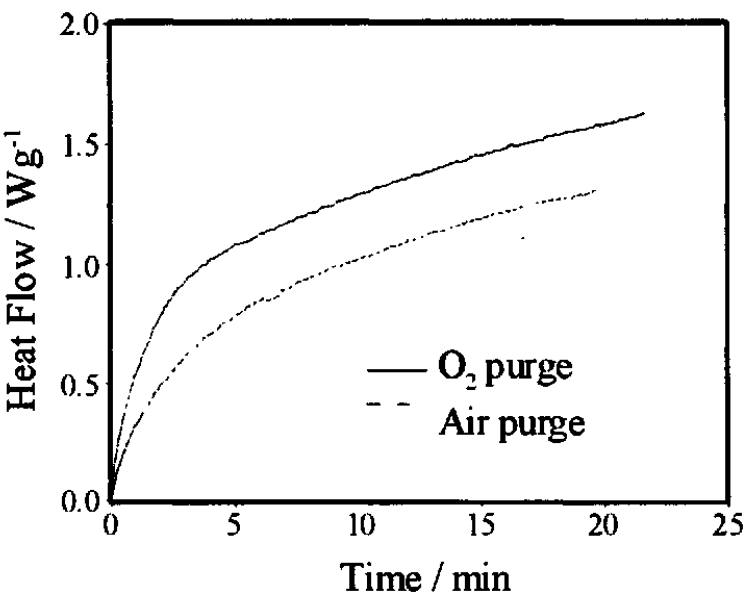

Figure 6 Photo-DSC exotherms for the PFMA-C ${ }_{60}$ film, prepared from $20 \mathrm{wt} \%$ PFMA and $1 \times 10^{-3} \mathrm{M} \mathrm{C}_{60}$ in diglyme, in $\mathrm{O}_{2}$ purge (solid line) and air purge (dotted line). Sample weight $=7.70 \mathrm{mg}$ and light intensity $=30.02$ $\mathrm{mW} / \mathrm{cm}^{2}$.

generate singlet oxygen in contrast to ordinary sensitizers. This explains the reason why the photo-curing of furan rings proceeds beyond an insolubilization point as long as the $\mathrm{C}_{60}$ continues absorbing photons and transferring the energy to the oxygen molecules. Only the self-oxidation of $\mathrm{C}_{60}$ terminates the photo-curing process.

\section{Conclusions}

The reaction mechanism for the POP resin was experimentally studied. The influence of post-exposure baking (PEB) and the effect of the oxygen partial pressure on the photo-curing behavior of the POP resin during UV light irradiation were investigated. Contrary to the PRP resin, PEB improved the sensitivity of the POP resin. Investigation of degrading the FAc endoperoxide by ${ }^{\mathrm{l}} \mathrm{H}$ NMR measurement afforded the activation energy and lifetime at $60^{\circ} \mathrm{C}$ to be $22.5 \mathrm{kcal} / \mathrm{mol}$ and $60 \mathrm{sec}$, respectively. Due to the thermal stability of the precursor at the crosslinkable point, a PEB process is necessary to cure the POP resin of this kind. While the photo-radical polymerization (PRP) resin showed a decrease in the thickness of developed film and the advent of reciprocity law failure at low intensity with increasing oxygen pressure, the POP resin showed no such phenomena. Moreover, the sensitivity of the POP resin was improved with increasing $\mathrm{O}_{2}$ pressure. The monotonically increasing exotherm curve in the photo-DSC chart for the POP resin suggested that $\mathrm{C}_{60}$ continuously produces singlet oxygen and is suitable for use as a sensitizer. 


\section{Acknowledgement}

We acknowledge the JSR Co. for supplying some reagents and the TA Instruments $\mathrm{Co}$. for the photo-DSC measurement support of this work. We also thank Dr. Y. Shigemitsu, Mr. E. Takeuchi and Mr. M. Miyazaki of the Institute of Physical and Chemical Research (RIKEN) for their experimental help and advice.

\section{References and notes}

1. A. Gandini and M. N. Belgacem, Prog. Polym. Sci. 22, 1203-1379, 1997

2. Y. Tajima, H. Arai, Y. Tezuka, T. Ishii and $\mathrm{K}$. Takeuchi, Fullerene Sci. and Technol. 5(7), 1531-1544 (1997).

3. Shinsozai purosesu sougongijutsu, 493,(1987).

4. Y. Tajima and K. Takeuchi, J. Photopolymer Sci. and Technol. 1998, 37-40.

5. H. Arai, Y. Tajima and K. Takeuchi J. Photopolymer Sci. and Technol. 12, 1, 1999, 121-124.

6. Y. Tajima, H. Arai, K. Takeuchi Kagaku Kogaku Ronbunshu 25, 6, 873-877 (1999).

7. A. A. Krasnovsky, Jr. Photochem. Photobiol. 1979, $29,29$.
8. E. Takeuchi, Y. Tajima, Y. Shigemitsu, K. Takeuchi and T. Hosomi J. Photopolymer Sci. and Technol. to be published.

9. Y. Tajima, Y. Shigemitsu, H. Arai, W. He, E. Takeuchi and $\mathrm{K}$. Takeuchi J. Photopolymer Sci. and Technol. 12, 1, 1999, 125-128.

10. M. E. C. Graupera, R A. Brito, N. Davidenko and P. O. Toro, An. Quim., 88, 294-296, 1990.

11. M. T. Beck and G. Mándi Fullerene Sci. and Technol. 5(2), 291-310 (1997).

12. J. W. Arbogast, C. S. Foote and M. Kao J. Am. Chem. Soc. 1992, 114, 2277-2279.

13. O. Ito Res. Chem. Intermed., 23(5), 389-402, 1997.

14. furfuryl acetate, ${ }^{1} \mathrm{H}$ NMR( $\left.\mathrm{C}_{6} \mathrm{D}_{5} \mathrm{CD}_{3}\right) \delta 1.57(\mathrm{~s}$, $3 \mathrm{H}), 4.83(\mathrm{~s}, 2 \mathrm{H}), 5.98(\mathrm{t}, J=3.3 \mathrm{~Hz}, 1 \mathrm{H}), 6.10(\mathrm{~d}$, $J=3.3 \mathrm{~Hz}, 1 \mathrm{H}), 6.99(\mathrm{t}, J=0.9 \mathrm{~Hz}, 1 \mathrm{H})$; furfuryl acetate endoperoxide, ${ }^{1} \mathrm{H}$ NMR $\left(\mathrm{C}_{6} \mathrm{D}_{5} \mathrm{CD}_{3}\right) \delta 1.55(\mathrm{~s}$, $3 \mathrm{H}), 4.39(\mathrm{dd}, \mathrm{J}=12.6,19.8 \mathrm{~Hz}, 2 \mathrm{H}), 5.52(\mathrm{~s}, 1 \mathrm{H})$, $5.70(\mathrm{~d}, \mathrm{~J}=5.7 \mathrm{~Hz}, \mathrm{H}), 5.95(\mathrm{~d}, \mathrm{~J}=5.7 \mathrm{~Hz}, 1 \mathrm{H})$

15. K. D. Ausman and R. B. Weisman Res. Chem. Intermed., 23(5), 431-451, 1997.

16. S. C. Clark, C. E. Hoyle, S. Jönsson, F. Morel and C. Decker Polymer 40, (1999) 5063-5072. 